This item was submitted to Loughborough's Research Repository by the author.

Items in Figshare are protected by copyright, with all rights reserved, unless otherwise indicated.

\title{
Confinement effects of levitons in a graphene cosmology laboratory
}

PLEASE CITE THE PUBLISHED VERSION

http://dx.doi.org/10.1039/C4RA11227J

PUBLISHER

(C) Royal Society of Chemistry

VERSION

VoR (Version of Record)

\section{PUBLISHER STATEMENT}

This work is made available according to the conditions of the Creative Commons Attribution 3.0 Unported (CC BY 3.0) licence. Full details of this licence are available at: http://creativecommons.org/licenses/by/3.0/

\section{LICENCE}

CC BY 3.0

\section{REPOSITORY RECORD}

Forrester, D. Michael. 2019. "Confinement Effects of Levitons in a Graphene Cosmology Laboratory”. figshare. https://hdl.handle.net/2134/16547. 


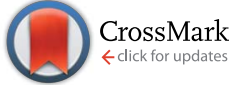

Cite this: RSC Adv., 2015, 5, 5442
Received 25th September 2014 Accepted 12th December 2014

DOI: $10.1039 / c 4 r a 11227 j$

www.rsc.org/advances

\section{Confinement effects of levitons in a graphene cosmology laboratory}

\begin{abstract}
Derek Michael Forrester*
The leviton, a long-lifetime soliton that rides the Fermi sea, is described here for a graphene system with a potential barrier. A full description of the leviton into the barrier is given for different angles of incidence. This is achieved by analysing the probability density of the leviton quasiparticle as a function of time. The transmission of the wavepacket through the oblique barrier occurs with negative refraction for low ratios of leviton energy to gate potential. In moderately strong magnetic fields a levity vortex becomes localised in space outside the barrier forming interior vortex states as it circulates. Also, when the field is switched on with an anti-leviton already formed inside the barrier, the quasiparticle becomes trapped and flows along its channel. Thus, the graphene system with leviton/anti-leviton propagation into and inside a barrier is a metamaterial whereby the direction of the quasiparticle can be controlled at will through the angle of the barrier and timing of switching on and off the magnetic fields. We present this analysis of barrier penetration for the inception of leviton electronics in graphene. The results lead to the possibility to create the graphene systems with levitons to explore cosmological questions such as hidden or "dark" energy conditions and therewith may also give some clues for understanding the discrepancies occurring between observed energy levels in the Universe and that of prediction.
\end{abstract}

\section{Introduction}

Early in the development of soliton science most people believed that at best these special waves were just a novelty and at worst an oddity with no real practical use. Some might even say that there was a degree of hostility to their existence, given the accounts of the history of this kind of wave motion. ${ }^{1}$ The observation of waves of translation, as solitons were originally called, was at odds with the mathematics of Stokes and Airy, and was made by Scott-Russell in $1834 .^{2}$ In the 1870's the theory of these water waves was derived by Boussinesq in 1871 (ref. 3) and by Rayleigh in $1876 .{ }^{4}$ Later, in 1895 , Korteweg and de Vries ${ }^{5}$ made the nonlinear partial differential equations to describe the Scott-Russell experiments. The interest in solitons from the scientific community waned after this and did not reignite until the significant findings of 1954 by Fermi, Pasta, Ulam and Tsingou (FPUT) for a one-dimensional lattice model using numerical experiments. ${ }^{6,7}$ In analysing the dynamics of coupled non-linear oscillators they found that energy initially diffused to other normal modes from the initial excited vibrational mode, but then returned to it, counter to the expected statistical spreading. In the discrete FPUT lattice the demonstration of energy sharing amongst only the lowest order modes prompted a continuum approximation and a study of the Korteweg-de Vries (KdV) equation by Zabusky and Kruskal in 1965 that

Departments of Chemical Engineering and Physics, Loughborough University, Leicestershire, UK. E-mail: d.m.forrester@lboro.ac.uk explained the physics also through numerical experiments. ${ }^{\mathbf{8}}$ The KdV equation was little understood until then and stable long-lifetime pulses were described only in detail after this seminal work.

The solitary waves are remarkable in that they preserve their shape over large distances and when two solitons collide they keep their integrity and speed. Nowadays solitons are essential to modern research. The examples of solitons in science range from self-cohering, localised excitations of a DNA polymer chain ${ }^{9}$ to dissipative solitons for mode-locked lasers. ${ }^{\mathbf{1 0}}$ In our work we are interested in a new near-soliton quasiparticle excitation that rides atop the Fermi sea. In 2013 Dubois et al. produced the first experimental verification of the existence of levitons, ${ }^{11}$ as predicted by Levitov and co-workers in the 1990s. ${ }^{12-14}$ The remarkable work of Dubois and co-workers was to create a single electron through voltage-pulse generation that appears out of the depths of the available energy states and travels as a soliton at the Fermi level. ${ }^{11,15}$ Using a sum of Lorentzian shaped voltage pulses the leviton emerged without any holes as the barrier height was modulated. In an earlier work we described the leviton in magnetic fields as it negotiates a potential step in a graphene system. ${ }^{16}$ Once the leviton has formed it can move into a potential step with almost perfect transmission when the leviton energy to potential step energy ratio is less than $0.28 .^{16}$ In low magnetic fields Young and Kim revealed experimentally that perfect transmission, Klein tunnelling, ${ }^{17}$ is a real phenomenon. ${ }^{18}$ For these reasons we primarily focus on a ratio of 0.27 , unless otherwise stated, in 
this paper as we investigate the levitons propagation into an oblique barrier in graphene. Graphene is a single atomic thick layer of graphite ${ }^{\mathbf{1 9 - 2 1}}$ (the material of pencil lead - the first graphene sheet was obtained by exfoliation of highly orientated pyrolytic graphite with sticky tape ${ }^{22,23}$ ) and has many amazing properties including electrons moving relativistically at an effective speed of light. ${ }^{24,25}$ We also consider the levity vortex as it forms in moderately strong magnetic fields and the revivals that occur as it rotates. We will then discuss the controlled propagation of levitons and anti-levitons in and around the potential barrier, wave-guiding phenomena, confinement effects, the consequences for electronic device design and an insight into possible vanishing or missing energy cosmological concerns. Thus, we address four thematic questions associated with the propagation of a leviton into a graphene potential barrier throughout the text: (1) "Can we switch on and off the Klein tunnelling in a potential barrier?", (2) "What happens to the leviton when we apply a magnetic field under the Klein tunnelling condition?" Upon answering this question and finding how to manipulate the trajectory of the quasiparticle, we then ask (3) "Can we completely confine a leviton or anti-leviton inside a potential, thus rendering it invisible?" In finding the answer to be yes, we foresee a new wave of possibilities for leviton electronics, but we also entertain the following speculative notion where we begin to ask, (4) "Can these phenomena be applied to cosmological mysteries?" Observations of the Universe in recent times have led to the belief that it continues to expand at an accelerated rate. ${ }^{26} \mathrm{~A}$ mysterious repulsion called dark energy seems to cause space to stretch ${ }^{27}$ Space is also filled with magnetic fields and magnetic tension between field lines has a tendency to flatten it. ${ }^{28}$ Thus, magnetocurvature is an important aspect to be considered in developing understanding of the Universe. Indeed Tsagas found that a region of magnetised space has a preference for a flat geometry due to the coupling between geometrical curvature and magnetism. ${ }^{28}$ The magnetic field need not even be comparatively strong for the tension of the field lines to have an effect when the curvature of space is large. In this paper we investigate the effects of magnetic fields in the seemingly two dimensional material of graphene and their influence on a newly discovered quasiparticle called the leviton. The graphene material is ideal to form an artificial laboratory for cosmological events due to the relativistic nature of its quasiparticles. Much of the Universe is inherently invisible and we explore how to create a veil around a leviton. Cosmology and particle physics are dictated by searches for missing particles, matter and energies. As such discoveries of the influences of potential barriers on particle propagation are highly relevant for conundrums such as dark energy, neutralinos, and galaxy formation.

For example, as the leviton propagates it spreads out such that its probability density decreases. ${ }^{16}$ If we imagine that it started point-like, such as whatever was at the beginning of the Universe, and it exploded into motion and expansion then it would have a particularly long lifetime. ${ }^{\mathbf{1 6}}$ As it is, in our leviton in graphene lab, its nature as a near-soliton makes it already exceptionally long lived, with enhancements due to confinement effects as we will see. So there may be analogy between a single neutrino left over from the Big Bang and the leviton in that it has been hypothesised that huge neutrino quantum wave functions stretch across billions of light years. ${ }^{29}$ It is an interesting question of quantum mechanics as to what the manner of the collapse of this wavefunction might be and if it distorts in the space-time curvature and what the influence of a magnetic field might be. Thus, we state the case that electronics including levitons could have a significant future role in helping us understand larger cosmological effects.

\section{Levitons into a $n-p-n$ barrier in a small magnetic field}

To answer these questions we need to characterise the movement of the leviton into a potential barrier so that we can understand the basic dynamics. We begin by considering the leviton as it moves in a small magnetic field of strength $B=5$ $\mathrm{mT}$, striking an oblique barrier that is inclined at $\theta$ degrees from the $y$-axis. The set-up is shown schematically in Fig. 1 as the leviton moves with Fermi velocity, $\nu_{\mathrm{F}} \approx 10^{6} \mathrm{~m} \mathrm{~s}^{-1,30}$ towards the barrier. The leviton has energy $E=0.27 U_{\mathrm{p}}$ with the potential barriers energy being $54 \mathrm{meV}$. The barrier is $156 \mathrm{~nm}$ wide. The system is characterised by a magnetic length $l_{B}=\sqrt{h / e B}$, where $B$ is the magnetic flux density. The dimensionless form of the Dirac equation for graphene in a magnetic field is given by, ${ }^{\mathbf{1 6}}$

$$
-i \partial_{\tau}\left(\begin{array}{l}
\Psi_{1} \\
\Psi_{2}
\end{array}\right)=\bar{H}\left(\begin{array}{l}
\Psi_{1} \\
\Psi_{2}
\end{array}\right)
$$

and

$$
\bar{H}=\left(\begin{array}{ll}
0 & -i \partial_{\bar{x}}-\partial_{\bar{y}}-\bar{y} B \\
-i \partial_{\bar{x}}+\partial_{\bar{y}}-\bar{y} B & 0
\end{array}\right)
$$

The dimensionless time is $\tau=\nu_{\mathrm{F}} t / 26 \mathrm{~nm}, \bar{U}_{\mathrm{p}}=26 \mathrm{~nm} / r \sqrt{B}$ and the coordinate positions are $\bar{x}=x / 26 \mathrm{~nm}$ and $\bar{y}=y / 26 \mathrm{~nm} .^{16}$ As the leviton moves through the system it takes the height and width of a Gaussian wavepacket. When it encounters the

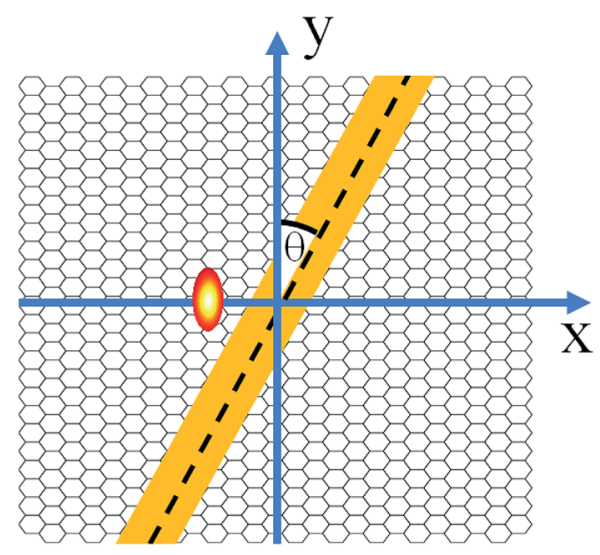

Fig. 1 (Colour online) After formation the leviton travels across the graphene layer until it meets a square potential barrier. The oblique potential barrier has size $U_{p}$ and is inclined at $\theta$ from the $y$-axis. 
potential barrier the probability density peaks upon it striking at incidence. Inside the barrier the anti-leviton forms. In Fig. 2
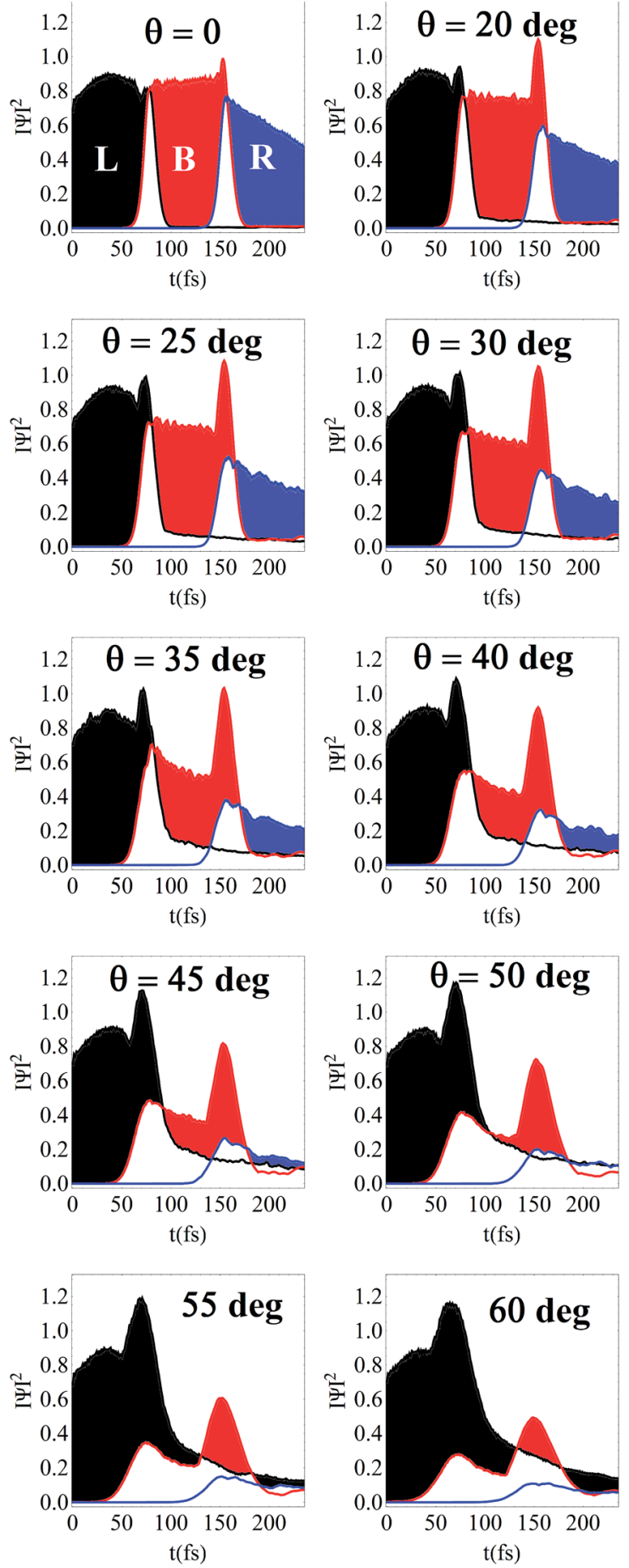

Fig. 2 (Colour online) The maximum probability density to the left (black lines and shading - L) and right (blue - R) of the barrier, and inside the barrier (red - B). The coloured shading indicates where the probability density is highest, but where there is no shading it indicates that the wavefunction has a strong presence in two or more regions. The angle $\theta$ indicates the angle of the barrier as shown in Fig. 1. The barrier is $156 \mathrm{~nm}$ wide with height $54 \mathrm{meV}$. The leviton has energy 0.27 times the barrier potential. we show the maximum probability density as a function of time in the regions prior to the barrier, inside the barrier and as a leviton leaves it on its far side. The leviton initially moves from left of the barrier. Fig. 2 charts the evolution of the wavefunction for different angles $\theta$. When $\theta$ is zero, then transmission is complete and Klein tunnelling occurs. There is even very little backscatter from the barrier up until about $\theta=20^{\circ}$.

Let us first discuss the case of Klein tunnelling at $\theta=0^{\circ}$ to understand the angular dependence. Fig. 2 shows three regions in each plot as mentioned and it is clearest for interpretation to start from $\theta=0^{\circ}$ where one can see that there is an overlap region (first white region between the red and black zones) whereby the leviton is right at the edge of the barrier and poised to cross into it. This balance point gives the peak in the maximum probability density at the left-hand edge of the barrier (peak of white intersection between black and red curves between $\mathrm{L}$ and $\mathrm{B}$ in Fig. 2). Then in the $\theta=0^{\circ}$ plot the antileviton forms in region $\mathrm{B}$ of the barrier and propagates without backscatter (or at least, not discernible levels - see ref. 16 for further insights) even when it meets the right-hand edge of the barrier. This is again marked in the plot by an intersection between the maximal probability densities in the barrier and that on the right of the barrier at the anti-leviton/leviton transition when the wavepacket sits right on the boundary between the two. This peak indicates that there is a confinement effect as the wavepackets "press" into the barrier edge, even though transmission ultimately becomes complete. Right of the barrier a leviton then reforms and continues on its path. It is important to note here that the levitons and anti-levitons are near-solitons and dissipate as they propagate, hence the spreading of the wavefunction seen in Fig. 2 by the reduction in the probability density with time. As mentioned above, the backscattering from the leading and trailing edge of the barrier is quite low until we reach higher angles of $\theta$. At $\theta=20^{\circ}$ one can clearly see that the probability density peaks at the edges of the barrier when the leviton is balanced half-in, half-out of the barrier. From the case of $\theta=0^{\circ}$ one can see that the leading edge probability density is smaller than at the trailing edge. At $\theta=20^{\circ}$ this is also so and this remains true until at $\theta=35^{\circ}$ the two become almost equal. As the angle of the oblique barrier is increasing there is more backscatter occurring from the lefthand edge. The transmitted rays negatively refract inside the barrier. Thus, the angle of approach of a leviton to a barrier is the first control parameter to negate Klein tunnelling when the leviton energy conditions are right with respect to the barrier height. Fig. 3 shows the evolution through the barrier at $\theta=45^{\circ}$ over a duration of 546 fs with $\nu_{\mathrm{F}} \approx 10^{6} \mathrm{~m} \mathrm{~s}^{-1}$ - one can see the large reflection of the leviton at this angle. Eventually, in Fig. 2 the reflected component of the wavefunction is completely dominant at $\theta=60^{\circ}$ and the probability density at the righthand edge of the barrier is hugely diminished in comparison to the preceding cases. The peak in the probability density (e.g. the red hump at $\theta=60^{\circ}$ in Fig. 2) completely disappears at $\theta=70^{\circ}$. In this case one finds that the leviton skims along the left-hand side wall of the barrier with such minute penetration that it is not visible in the probability density evolution plots. Thus, we end this section having described the dynamics of the 

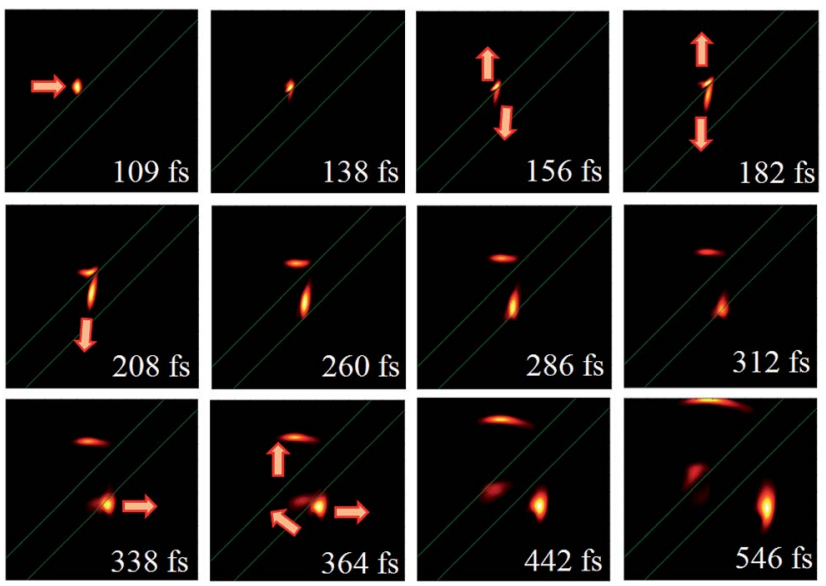

Fig. 3 (Colour online) The absolute value of the probability density of the leviton/anti-leviton negotiating the barrier which is inclined at $\theta=45^{\circ}$. The barrier is $156 \mathrm{~nm}$ wide with height $54 \mathrm{meV}$. The leviton has energy 0.27 times the barrier potential.

leviton into a typical potential barrier with different angularity. Next we increase the magnetic field.

\section{Levity vortex formation and confinement in moderate magnetic fields}

Now if we increase the magnetic field to $2.5 \mathrm{~T}$ we expect the magnetic field to bend the trajectory of the quasiparticle into the barrier. What actually happens is that the leviton becomes localised into a continuous anti-clockwise orbit outside the barrier with this sufficiently high magnetic field. ${ }^{16}$ We call this rotating quasiparticle a levity vortex. There exist localised pockets of zero probability density inside that levity vortex as it evolves in time. In Fig. 4 the vortex is rotating adjacent to the barrier but with minor interaction. At $39 \mathrm{fs}$ the rotation of the vortex generates a region in its core that remains devoid of any presence of the leviton. At 117 fs a second "cold spot" forms in the vortex interior. The vortex continues to be populated by such localised zero probability density states within its interior. When the levity vortex forms it warps as it rotates and can trap a vortex bound state. We speculate that these trapped states can lead to the formation of Majorana fermions akin to those recently found in superconductors. ${ }^{31}$ The circulation of the levity vortex gives rise to trapped zero-energy modes at the barrier edge. This occurs as the levity vortex circulates and with each $2 \pi n$ rotation another bound state is potentially created. In this condition the leviton forms a superposition state with an anti-leviton, hence creating the zero energy bound state where the leviton is its own anti-particle. Interestingly the levity vortex also has an enhanced lifetime and undergoes a series of revivals as it circulates (see, Fig. 4). The presence of the barrier is important because at its edges the leviton and anti-leviton are in a state of crossover marked by an enhancement in the amplitude of the probability density, as we saw in Fig. 2. We have also seen that there is some delay time into the barrier and hence
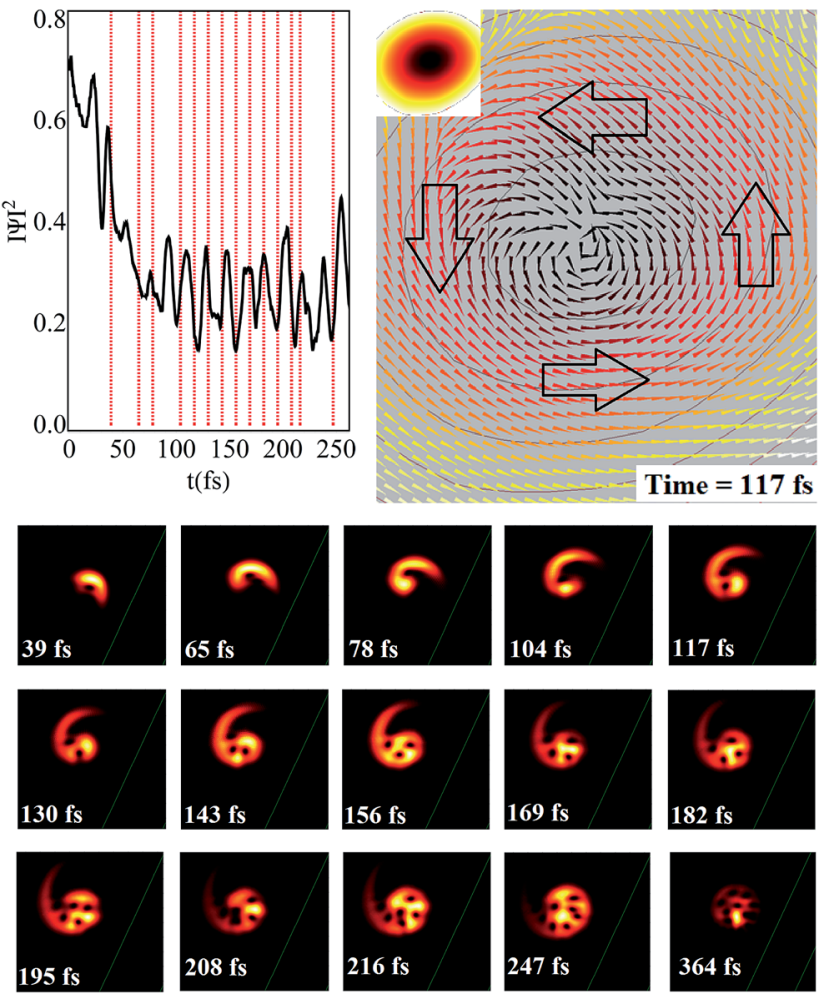

Fig. 4 (Colour online) Outside the barrier a leviton becomes trapped in a cyclotronic orbit as a result of increasing the magnetic field to $B=$ $2.5 \mathrm{~T}$. On the top left, a plot of the maximum probability density as a function of time is shown. There are a series of revivals of the wavefunction. In the snap-shots of the circulating leviton, which we call a levity vortex, one can see that upon rotation of the levity vortex new "cold spots" emerge (black dots). The maximum probability density in these snap-shots is shown in yellow, intermediate levels by red and zero by black. Each "cold spot" is a vortex that penetrates the levity quasiparticle. The interior vortices rotate anti-clockwise and the top right image shows an arrow plot of a vortex that typically emerges in the system (shown for $117 \mathrm{fs}$ ).

prolonged duration of superposition at the edges were Majoranas can potentially form. The leviton is atop the Fermi sea and a continuous supply of left and right moving electrons support its existence and endurance. The magnetic field provides the impetus for the formation of the bound states at the vortex. The leviton exists in an unusual condition already and it may be that it becomes closer to forming Majorana like fermions due to existing without prior entanglement to a hole. Thus, increasing the magnetic field we find that the trajectory of the leviton becomes localised in space as a vortex. For small magnetic fields the leviton curves into the barrier, whilst upon switching the strength of the field upwards it becomes the levity vortex quasiparticle. This is another control parameter against Klein tunnelling. In Fig. 5 it can be seen that the levity vortex is easily trapped when surrounded by a closed boundary potential. Two cases of system energies are shown; one at $E_{0}=0.33 U_{\mathrm{p}}$ and the other at $E_{0}=0.93 U_{\mathrm{p}}$. Both are in a $6.5 \mathrm{~T}$ magnetic field. In the former there is some degree of penetration into the barrier as the levity vortex circulates, but no emission into the outer environment. For the latter leviton energy there is no 

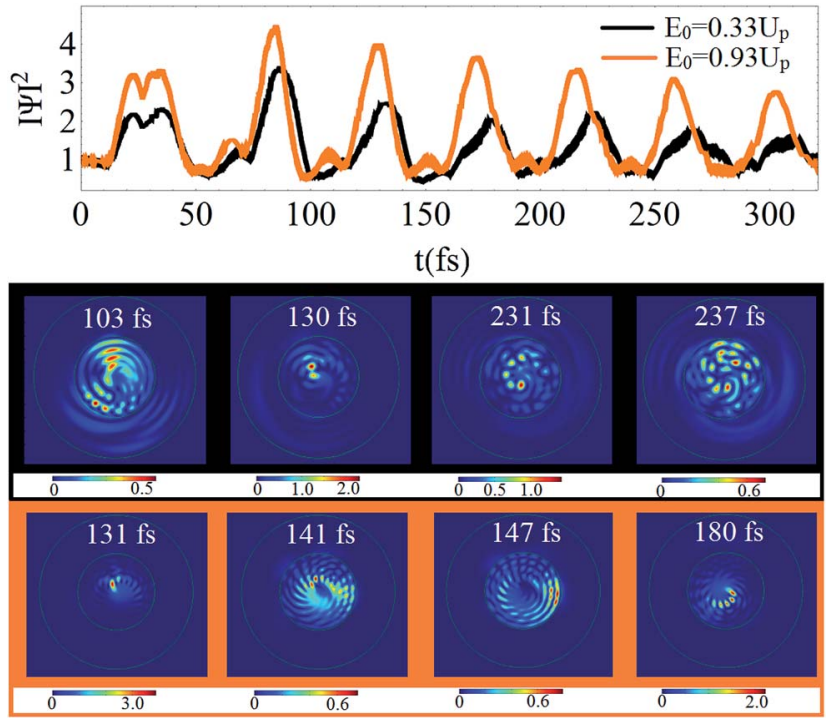

Fig. 5 (Colour online) The leviton forms surrounded by a circular potential barrier, with $E_{0}=0.33 U_{\mathrm{p}}$ (black) and $E_{0}=0.93 U_{\mathrm{p}}$ and a $6.5 \mathrm{~T}$ magnetic field. Underneath the probability density plot the evolutions of the leviton for these two cases are shown, respectively.

propagation into the barrier at all. Thus, the levity vortex is completely screened from the outer environment. Comparing Fig. 5 against Fig. 4 one can note that the probability density is much higher due to the increased confinement of the leviton in a circular potential in contrast to residing in close proximity to a linear barrier. Therefore, we can hide the leviton inside the potential barrier in a fashion that could be perceived as analogous to the situation of a black hole. The leviton can be released by lowering the magnetic field and/or the potential barrier. After entrapment the deconfinement process involves lowering the potential such that $E_{0} / U_{\mathrm{p}}>1$. Then as the levity vortex rotates it seeps through the space occupied by the circular barrier and in the outer environment begins its own rotational cycle. Lowering the magnetic field will also expand the radius of this rotation.

\section{Confinement inside the oblique barrier}

Now that we have determined what happens to the leviton outside the oblique barrier in an applied magnetic field, we consider the effects associated with ramping up the strength of the magnetic field when the anti-leviton is firmly formed inside the barrier. For this scenario the magnetic field is smoothly increased from its initial level with a maximum occurring at half way inside the barrier. Recall that in a small magnetic field of $5 \mathrm{mT}$ that there is perfect transmission when the leviton approaches with $\theta=0$ (Fig. 2). Now allowing the magnetic field to remain at $5 \mathrm{mT}$ till the anti-leviton has formed inside the barrier and then increasing the magnetic field smoothly up to $2.5 \mathrm{~T}$, one manages to reverse the trajectory so that it does a complete "U-turn". Inside the barrier the anti-leviton moves in a clockwise direction at this strength of field. This can be seen in Fig. 6. If the field is increased to $8 \mathrm{~T}$ rather than $2.5 \mathrm{~T}$ then the

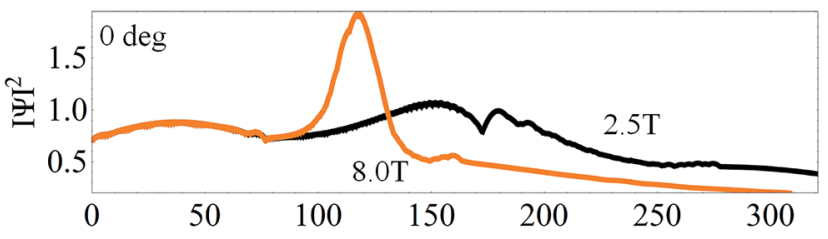

$\mathrm{t}(\mathrm{fs})$
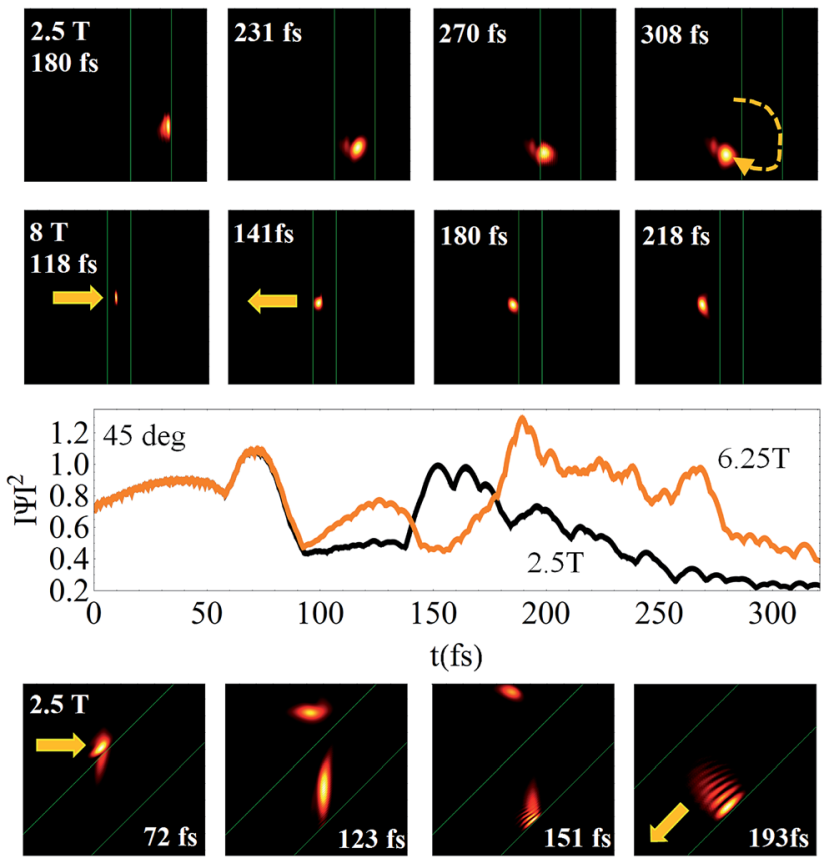

Fig. 6 (Colour online) In the top plot the probability density is shown as a function of time when $\theta=0$ and the magnetic fields are increased from $B=0.05 \mathrm{~T}$ to $2.5 \mathrm{~T}$ (black) and 8.0 T (orange) when the propagating quasiparticle has entered the barrier. In the second row the evolution of the leviton into the barrier is shown at an increase to $2.5 \mathrm{~T}$. A rotated trajectory is shown to occur and is indicated by the dashed arrows in the snapshot for $308 \mathrm{fs}$. In the third row the field was increased to $8 \mathrm{~T}$. The arrows in the snapshots indicate the direction of the trajectory. The fourth row shows $|\Psi|^{2}$ for the similar change in field strength to the above scenarios but for an oblique barrier with $\theta=45^{\circ}$. The final row shows the trapping of the "ray" inside the potential.

leviton does not carry out a gentle about-turn, but is instead batted straight back along the direction from which it came (see Fig. 6). Therefore, the increasing and decreasing of the applied magnetic field can be used to control the direction of the wavepacket and can be programmed accordingly. Thus, pulsed sequences of magnetic field can bounce the leviton backwards and forwards like a tennis ball or make it change direction like a heavy moving vehicle (at lower field strengths).

The next stage is to see what happens when the angle of the barrier is increased. In this case we can trap the anti-leviton inside the barrier. In Fig. 6 this is demonstrated for $\theta=45^{\circ}$ and field strengths of 2.5 and $6.25 \mathrm{~T}$. The effect is the same for both field strengths: the leviton is allowed to enter the barrier as was the situation in Fig. 3, except in this case the magnetic field is increased when the anti-leviton has formed. This completely traps the quasiparticle and it travels down the barrier as if it were a motorway. Anti-levitons are transmitted along the 
interior of the p-junction boundary without external scattering when the confinement effect, generated by magnetic field manipulation, is operational. Such confinement may have far reaching implications because without radiating out of the potential it means that the internal dynamics can be hidden from view. For example, if a "ray" strikes a potential in the manner of Fig. 5 then partial reflection may ensue but the rest of the wavepacket becomes trapped. On a grander scale this means that if there exist regions of various high potential energies in the Universe that they can ensnare a large amount of mass. A candidate for where such events could be happening is at cosmic strings. Thus, the graphene levitons may be used to gleam insights into many other analogous systems and perhaps answer some profound mysteries.

\section{Discussion and conclusions}

We have answered our four questions of the introduction and discovered how to control the movement of a leviton outwith and inside a potential. As we have seen, the leviton rides the Fermi sea and at the edges of the potential begins a transition into an anti-leviton. At these edges and possibly within the core of a levity vortex, zero energy states can emerge. This is marked by a large increase in the probability density at the edges. In these regions, at the barrier, there can be the highest localised concentration of levitons/anti-levitons and the soliton can be in a superposed state of the two. This means that there is the possibility for the existence of electrically neutral Majorana fermions in these regions in a graphene system.

For many years graphene systems have been touted as possible playgrounds to begin to understand cosmological problems in the laboratory. One of the greatest mysteries is where and what the unaccounted for $95 \%$ of matter and energy in the Universe is. ${ }^{32}$ In some theories of cosmology, it is estimated that there is a cosmic string ${ }^{33-35}$ every $10^{31}$ cubic light years in the Universe. Each of these strings is a large potential at which it may be possible that, like in graphene barriers, there are existing Majorana-like particles. With charge neutrality they would remain invisible to electromagnetic detection and, as we have noted for graphene, the largest probability amplitudes exit where a confinement effect is occurring. Thus, at a transition through a barrier, or a string, there is a concentration of Majorana particles. However, one would then expect to locate them after the delay time at the barrier as the anti-particles are created in the barrier before emission at the far side or as a reflected pulse at the initial side of the potential. But the current work has demonstrated that a potential under the right conditions can hide their existence. We found this when we increased the magnetic field strength as the anti-leviton was fully formed within the barrier. We also showed that a potential can be used to completely screen even the most energetic of motions in the case of the levity vortex. When the ratio of the quasiparticle energy to that of the potential is low enough that transmission is almost complete this has been demonstrated, whereas at higher ratios then there is a steady increase in reflection. ${ }^{\mathbf{1 6}}$ For relativistic particles this means that potential barriers will act as selective filters for different particle energies. Here the angle of the barrier is also of primary importance with respect to the trajectory of the soliton. With this in mind, it seems possible that we would be unable to account for large quantities of the expected matter and energy in the Universe. The wavepacket can become trapped in a potential and the potential becomes nonradiating. There is also another possibility for the missing mass in the Universe and that is that there are many potential barriers, such as cosmic strings or the like, that can be acting as effective cloaks of invisibility to a host of matter. Thus, we put forward the concept that perhaps the Universe has a design whereby naturally we cannot detect what lies inside regions of high potential because of this confinement effect, which may also be contributed to by the emergence of Majoranas. It is perhaps possible that because there is an energy filtering effect that occurs, then there are regions of space which will not be completely "dark" but instead bright to the observer, whilst simultaneously hiding a substantial amount of energy and matter in various potential trappings.

Beyond this concept, we assert that this new quasiparticle called a leviton has an enormous potential for use in electronics because it can be controlled into any trajectory by varying the strength of magnetic fields in conjunction with barrier positioning and amplitude. The propagation and trajectory of the levitons/anti-levitons is thus determined. The barrier can offer topological protection to the wavepacket through designs that are capable of raising and lowering its height. This could be very useful for quantum computing schemes where decoherence is a fundamental stumbling block to implementing practical logic operations. ${ }^{31,36}$ It could also be a useful condition for creating broadband filtering devices. However, it is now important for experiments to be performed to verify or refute the confinement effects we highlight. If they are realised in the laboratory then the implication is that the Universe is under a shroud that is disguising and hiding much of its secrets by the prevalence of potential energy defects, or the like, that can block many discoveries by their presence.

Field induced confinement effects in graphene were discussed by Moriyama et al. for an experimental system that demonstrated the Coulomb-blockade effect in a perpendicular, uniform magnetic field. ${ }^{37}$ The metallic/graphene junctions were used to induce an electrostatic surface-potential and magnetic fields generated quantum dots. Confinement and release of the Dirac particles was dictated by the magnetic field. These Dirac particles will still interact with their environment and therefore destroy the purity of the quantum state, whereas soliton-like levitons exist in a decoherence free form. ${ }^{38}$ The leviton is also a long-lived excitation that propagates over relatively large distances, even without careful incorporation of waveguides that boost their amplitude, e.g. with barrier/field induced quantum revivals. Thus, for the creation of clean electrical current pulses that are free from electron-hole pairs, charge pulses at a quantum point contact ${ }^{\mathbf{1 1}}$ are expected to facilitate the formation of levitons in the graphene system. The levitons are then readily channelled into any potential barrier. The emergence of levitons is verifiable through electronic Hong-OuMandel experiments. ${ }^{11}$ The design of gated structures on the graphene substrate can be carried out through lithography and 
features such as metal plates with circular holes built. ${ }^{39}$ Levitons of arbitrary width and pulse duration can be excited into existence from the Fermi-sea using micro-circuitry, eliminating the need to use advanced nanolithography methods and so creating a scalable design ethic. ${ }^{11}$ However, for quantum electronics using levitons the barrier widths should be of similar length to the magnetic length, $l_{B}{ }^{40}$ and contacts fabricated through electron beam lithography. Upon requirement of higher carrier mobilities freely suspended graphene sheets ${ }^{\mathbf{4 1}}$ could be incorporated. Etching the underlying substrate will also lead to avoidance of unwanted trapped charges and surface roughness effects. Alternatively graphene bubbles with a controllable curvature can be manipulated with electric fields and they also have high mobilities. ${ }^{\mathbf{4 2 , 4 3}}$ It is difficult to confine graphene carriers in a potential well without the addition of electric and magnetic fields due to the Klein tunnelling phenomenon. ${ }^{44}$ But it is also worthwhile to point out that Portnoi and co-workers have also reached the conclusion that complete confinement of charge carriers in graphene by electrostatic potentials can occur for zero-energy states, thus negating Klein tunnelling and allowing the creation of novel quantum dots and waveguides. ${ }^{45,46}$ It is because of confinement effects that a graphene leviton system could be an architypical prototype for a lab on a chip.

\section{Acknowledgements}

The author thanks the EPSRC for funding under KTA grant "Developing prototypes and a commercial strategy for nanoblade technology" and Professor Feodor Kusmartsev for discussion.

\section{References}

1 A. T. Filippov, Versatile Solitons, Springer, New York Dordrecht Heidelberg London, 2nd edn, 2010.

2 J. S. Russell, Report of the 14th Meeting of the British Association for the Advancement of Science, 1844, pp. 311-390.

3 M. J. Boussinesq, C. R. Acad. Sci., 1871, 72, 755-759.

4 J. W. S. L. Rayleigh, Philos. Mag., 1876, 1, 257-279.

5 D. J. Korteweg and G. de Vries, Philos. Mag., 1895, 39, 422443.

6 E. Fermi, J. R. Pasta and S. Ulam, Report, Los Alamos: Los Alamos Scientific Laboratory, LA-1940, 1955.

7 J. L. Tuck and M. T. Menzel, Adv. Math., 1972, 9, 399-407.

8 N. J. Zabusky and M. D. Kruskal, Phys. Rev. Lett., 1965, 15, 240.

9 L. L. V. Zandt, Phys. Rev. A, 1989, 40, 6134(R).

10 P. Grelu and N. Akhmediev, Nat. Photonics, 2012, 6, 84-92.

11 J. Dubois, T. Jullien, F. Portier, P. Roche, A. Cavanna, Y. Jin, W. Wegscheider, P. Roulleau and D. C. Glattli, Nature, 2013, 502, 659-663.

12 L. S. Levitov, H. Lee and G. B. Lesovik, J. Math. Phys., 1996, 37, 4845-4866.

13 D. A. Ivanov, H. W. Lee and L. S. Levitov, Phys. Rev. B: Condens. Matter Mater. Phys., 1997, 56, 6839-6850.
14 J. Keeling, I. Klich and L. S. Levitov, Phys. Rev. Lett., 2006, 97, 116403.

15 C. Flindt, Nature, 2013, 502, 630-632.

16 D. M. Forrester and F. V. Kusmartsev, Nanoscale, 2014, 6, 7594-7603.

17 M. I. Katsnelson, K. S. Novoselov and A. K. Geim, Nat. Phys., 2006, 2, 620-625.

18 A. F. Young and P. Kim, Nat. Phys., 2009, 5, 222-226.

19 P. R. Wallace, Phys. Rev., 1947, 71, 622-634.

20 X. Zhang, B. R. S. Rajaraman, H. Liub and S. Ramakrishna, RSC Adv., 2014, 4, 28987-29011.

21 J. Zhu, M. Chen, Q. He, L. Shao, S. Wei and Z. Guo, RSC Adv., 2013, 3, 22790-22824.

22 K. S. Novoselov, A. K. Geim, S. V. Morozov, D. Jiang, Y. Zhang, S. V. Dubonos, I. V. Grigorieva and A. A. Firsov, Science, 2004, 306, 666-669.

23 J. Kang, D. Shin, S. Baea and B. H. Hong, Nanoscale, 2012, 4, 5527-5537.

24 A. K. Geim and K. S. Novoselov, Nat. Mater., 2007, 6, 183-191. 25 K. S. Novoselov, A. K. Geim, S. V. Morozov, D. Jiang, M. I. Katsnelson, I. V. Grigorieva, S. V. Dubonos and A. A. Firsov, Nature, 2005, 438, 197-200.

26 M. Ishak, A. Peel and M. A. Troxel, Phys. Rev. Lett., 2013, 111, 251302.

27 S. Perlmutte, M. S. Turner and M. White, Phys. Rev. Lett., 1999, 83, 670 .

28 C. G. Tsagas, Phys. Rev. Lett., 2001, 86, 5421.

29 G. M. Fuller and C. T. Kishimoto, Phys. Rev. Lett., 2009, 102, 201303.

30 C. Hwang, D. A. Siegel, S.-K. Mo, W. Regan, A. Ismach, Y. Zhang, A. Zettl and A. Lanzara, Sci. Rep., 2012, 590.

31 V. Mourik, K. Zuo, S. M. Frolov, S. R. Plissard, E. P. A. M. Bakkers and L. P. Kouwenhoven, Science, 2012, 336, 1003-1007.

32 R. Caldwell and M. Kamionkowski, Nature, 2009, 458, 587589.

33 Y. B. Zeldovich, Mon. Not. R. Astron. Soc., 1980, 192, 663-667. 34 T. W. B. Kibble, Nature, 1985, 317, 472.

35 W. H. Zurek, Nature, 1985, 317, 505-508.

36 F. Wilczek, Nature, 2012, 486, 195-197.

37 S. Moriyama, Y. Morita, E. Watanabe and D. Tsuya, Appl. Phys. Lett., 2014, 104, 053108.

38 D. Ferraro, B. Rousse, C. Cabart, E. Thibierge, G. Féve, C. Grenier and P. Degiovanni, Phys. Rev. Lett., 2014, 113, 166403.

39 G. Giavaras, P. A. Maksym and M. Roy, J. Phys.: Condens. Matter, 2009, 21, 102201.

40 N. Gu, M. Rudner, A. Young, P. Kim and L. Levitov, Phys. Rev. Lett., 2011, 106, 066601.

41 K. I. Bolotin, K. J. Sikes, Z. Jiang, M. Klima, G. Fudenberg, J. Hone, P. Kim and H. L. Stormer, Solid State Commun., 2008, 146, 351-355.

42 T. Georgiou, L. Britnel, P. Blake, R. V. Gorbachev, A. Gholinia, A. K. Geim, C. Casiraghi and K. S. Novoselov, Appl. Phys. Lett., 2011, 99, 093103.

43 A. B. G. Trabelsi, F. V. Kusmartsev, B. J. Robinson, A. Ouerghi, O. E. Kusmartseva, O. V. Kolosov, R. Mazzocco, 
M. B. Gaifullin and M. Oueslati, Nanotechnology, 2014, 25, 45 C. A. Downing, D. A. Stone and M. E. Portnoi, Phys. Rev. B: 165704. Condens. Matter Mater. Phys., 2011, 84, 155437.

44 P. A. Maksym, M. Roy, M. F. Craciun, S. Russo, 46 R. R. Hartmann, N. J. Robinson and M. E. Portnoi, Phys. Rev. M. Yamamoto, S. Tarucha and H. Aoki, J. Phys.: Conf. Ser., B: Condens. Matter Mater. Phys., 2010, 81, 245431. $2010,245,012030$. 